\title{
EL NEOCOLONIALISMO EN NUESTROS DÍAS: LA PERSPECTIVA DE LEOPOLDO ZEA
}

\author{
Karla Cecilia Macías Chávez \\ doi:10.11144/Javeriana.uph32-65.nplz
}

\begin{abstract}
RESUMEN
El presente escrito busca contestar a dos preguntas: ¿Es la posición de Leopoldo Zea acerca del sistema neocolonialista de su tiempo obsoleta, respecto a la situación en que vivimos actualmente muchos habitantes de México y de otros países del mundo sujetos a un dominio neocolonial; o, es posible y adecuado pensar en su postura como una vía de crítica e incluso de propuesta de acción ante el panorama neocolonialista en nuestros días? ¿Por qué sostenemos una u otra postura? Para contestar a ambas preguntas definiremos y posicionaremos en un contexto histórico-teórico el término neocolonialismo. Además, expondremos algunas de las formas en las que se manifiesta este sistema de dominación en países latinoamericanos desde hace no mucho tiempo. Asimismo, ofreceremos algunos de los puntos más importantes que Zea consideraba al pensar, describir y explicar esta situación, y algunas posibles propuestas de reflexión y acción ante la misma.
\end{abstract}

Palabras clave: neocolonialismo; comprensión histórica; asimilación; toma de conciencia; diversidad

* Universidad Michoacana de San Nicolás de Hidalgo, Morelia, Michoacán, México Correo electrónico: kc.macias.chavez@gmail.com

Para citar este artículo: Macías Chávez, K.C. (2015). El neocolonialismo en nuestros días: La perspectiva de Leopoldo Zea. Universitas Philosophica, 32(65), pp. 81-106. ISSN 0120-5323, ISSN en línea: 2346-2426, doi:10.11144/Javeriana.uph32-65.ncplz 


\title{
NEOCOLONIALISM IN OUR DAYS: LEOPOLDO ZEA'S APPROACH
}

\author{
Karla Cecilia Macías Chávez
}

\begin{abstract}
This paper seeks to answer two questions: Is Leopoldo Zea's position on the neocolonialist system of his days outdated with regard to the situation many of us are living in Mexico and other countries in the world, subject to neocolonial domination right now; or is it possible and proper to consider his position as a way of criticism and even as an action proposal to the colonialist outlook nowadays? Why do we hold one position or another? To answer both questions the term 'neocolonialism' will be defined and positioned within a historical and theoretical framework. In addition, we will discuss some of the ways this system of domination in Latin American countries is recently manifested. Also, we will offer some of the most important insights Zea had into account when understanding, describing and explaining this situation, and some possible proposals for reflection and action into it.
\end{abstract}

Key words: neocolonialism; historical understanding; assimilation; awareness; diversity 


\section{Introducción}

EN EL PRESENTE ENSAYO se expondrán algunas de las ideas actuales y pasadas (s. XX) sobre una situación que ha estado vigente durante más de un siglo en México y en otros países de Latinoamérica, Asia y África: el fenómeno neocolonialista. Y esto, con el fin de relacionar algunas de las ideas y circunstancias más recientes que existen sobre este fenómeno en Latinoamérica con las ideas ya existentes sobre el mismo en el pensamiento de Leopoldo Zea. Específicamente, el propósito del ensayo es responder, con base en el análisis propuesto, a estas dos preguntas: ¿Es la posición de Zea respecto a este tema (neocolonialismo) obsoleta en cuanto a la situación que vivimos actualmente muchos habitantes de este y de otros países del mundo sujetos a un dominio neocolonial, o es pertinente considerarla seriamente como una alternativa de crítica, pensamiento e incluso de iniciativa de acción para tratar con la situación neocolonialista que muchos países padecen? ¿Cuáles son las razones para sostener lo anterior?

Es importante discutir este tema, ya que es una situación que vivimos día a día y que nos afecta de manera directa. Dado que estamos inmersos en ella, es necesario reflexionarla y criticarla para poder tomar, así, una posición sobre la misma. De tal forma, podremos buscar propuestas de solución y debate tanto en fuentes históricas como en ideas presentes. Este ejercicio crítico forma parte de una actitud ética que es ideal promover en cualquier sociedad, de manera que no nos comportemos como sonámbulos, y no actuemos inconscientemente ante la situación que nos rodea.

Para responder a la pregunta mencionada y cumplir con el objetivo del artículo, comenzaremos por situar el concepto "neocolonialismo" en su correspondiente contexto histórico, de forma que lleguemos a una definición concreta de lo que debe entenderse por este. En segundo término, se expondrán algunas de las ideas y situaciones más recientes que acontecen en torno a este fenómeno. A continuación, mencionaremos la relevancia, las implicaciones y las ideas que conciernen a este concepto en la visión de Leopoldo Zea. En cuarto lugar, contestaremos a las preguntas formuladas previamente basándonos en lo anteriormente expuesto. Finalmente, esbozaremos algunas conclusiones sobre el tema. 


\section{Neocolonialismo: contexto y definición}

Es PERTINENTE SITUAR el concepto "neocolonialismo" en el contexto que le corresponde, dado que se trata de un término que está íntimamente ligado a otros con los cuales podría entremezclarse y confundirse. Es imprescindible, por tanto, mencionar ciertos rasgos del panorama ideológico e histórico en el que se encuentra con el fin de entenderlo con cierta profundidad y, asimismo, comprender mejor la definición que se estará utilizando para criticar y analizar el fenómeno neocolonial a lo largo de este escrito.

En primer lugar, es importante resaltar las diferencias entre "neocolonialismo", "poscolonialismo" y "decolonialidad", tres conceptos que pueden ser, fácilmente, motivo de confusión. Estos términos forman parte de una serie de teorías y críticas que se han llevado a cabo principalmente durante el siglo XX. Por su lado, el término "poscolonialismo" puede ser entendido de distintas maneras dependiendo a lo que nos estemos refiriendo (Young, 2008). En el Oxford Dictionary se define como "ocurriendo o existiendo después del término del régimen colonial [traducción propia]" (Oxford Dictionary, "postcolonial”). El Collins Dictionary lo define como "existiendo u ocurriendo desde que una colonia ganó independencia” (Collins Dictionary, "post-colonial”. Traducción mía).

Por su parte, Robert J. C. Young, profesor de New York University, coincide en que poscolonialismo puede definirse como el periodo que viene después de un régimen colonialista o imperialista, en su significado original de dominación directa. Sin embargo, existen otras acepciones que se le han dado al término, entre las que encontramos la histórica. De acuerdo con esta, poscolonialismo se entiende como la suma de todos los hechos históricos que tuvieron lugar en el proceso descolonizador, en el que sobresalía la determinación de las antiguas colonias por alcanzar su soberanía. Determinación que trae como consecuencia la faceta cultural del concepto, que comprende, entre otras cosas, el surgimiento de pensamientos e ideas críticos resultado de la reflexión sobre la situación que se vivía, y sobre la legitimidad y pertinencia de los regímenes impuestos por países colonizadores.

Otro de los autores que trata directamente con la pluralidad de aspectos a los que el concepto de poscolonialismo hace referencia es Stuart Hall. Él considera que es difícil saber lo que significa exactamente y se refiere a este término como si 
estuviese en proceso de desaparición. Hall (1996) lo cuestiona desde distintas perspectivas y busca deconstruirlo; sin embargo, reconoce su importancia para la discusión de ciertos temas como el de identidad.

En la misma línea de la discusión a la que Hall refiere, existe también bastante literatura que se considera poscolonial, debido a que coincide con el espíritu crítico que surgió en muchas de las antiguas colonias y que cuestiona la autoridad de los potencias colonizadoras en sus distintos aspectos. A pesar de haber tanta literatura poscolonial, que algunos consideran existe desde hace mucho tiempo atrás, por ejemplo en los escritos de Las Casas en el S. XVI, no se había establecido como una escuela de pensamiento hasta el siglo XX. Por tanto, el poscolonialismo también se puede entender como una línea de pensamiento y crítica en disciplinas como la filosofía. El primero en inspirar el establecimiento de esta escuela fue el crítico y teórico palestino Edward Said a través de su obra Orientalism (1978). En esta, el autor muestra, entre otras cosas, sus ideas sobre los prejuicios que usualmente se tienen de Oriente, tales como la costumbre de caracterizarlo como mágico o místico y no precisamente racional. Dicha obra estableció la pauta para que se siguiera la crítica en esta línea, y fue así como algunas teorías fueron desarrollándose, de manera que se formaron grupos de intelectuales que apoyaban estas ideas (Young, 2006).

El Grupo de Estudios Subalternos, por ejemplo, fue fundado en los años 80 por Ranajit Guha, un historiador indio, influenciado por el pensamiento de Said. Este grupo, a su vez, fue la influencia principal para la formación del Grupo Latinoamericano de Estudios Subalternos en los años 90, el cual estaba constituido por John Beverly, Javier Sanjinés, Patricia Seed, Walter Mignolo, entre otros. Mignolo, más adelante, se integrará al Grupo Modernidad/Colonialidad (grupo MC), del que formarán parte personajes como Enrique Dussel, Aníbal Quijano y Santiago Castro-Gómez. Este último colectivo hizo grandes contribuciones a la reflexión de temas sobre el poscolonialismo, cifrando entre estas la introducción del concepto "decolonialidad".

Mignolo (2007, p. 27) explica que "el pensamiento decolonial emergió en la fundación misma de la modernidad/colonialidad como su contrapartida. Y eso ocurrió en las Américas, en el pensamiento indígena y en el pensamiento afro-caribeño; continuó luego en Asia y África”. El concepto "decolonialidad", producto de este particular pensamiento, es utilizado por el grupo MC en el 
sentido de giro decolonial y fue, además, desarrollado originalmente por el filósofo puertorriqueño Nelson Maldonado-Torres. Mignolo (2007) explica que la decolonialidad es:

la energía que no se deja manejar por la lógica de la colonialidad, ni se cree los cuentos de hadas de la retórica de la modernidad. Si la decolonialidad tiene una variada gama de manifestaciones (...), el pensamiento decolonial es, entonces, el pensamiento que se desprende y se abre (...), encubierto por la racionalidad moderna, montado y encerrado en las categorías del griego y del latín y de las seis lenguas imperiales europeas modernas. (p. 27).

La descolonización, tal como la encontramos en el Diccionario de la Real Academia Española, refiere a la "[s]upresión de la condición colonial de un territorio" (RAE, "descolonización"). Sin embargo, esta definición no alcanza a abarcar las especificidades que conlleva la "verdadera" supresión de tal régimen en todas sus facetas. Es decir, según los intelectuales del grupo MC, el pensamiento decolonial debe complementar o completar la descolonización que se llevó a cabo en los siglos XIX y XX, lo cual quiere decir que no es suficiente que exista una independencia política o económica, también es necesario "descolonizar" el pensamiento, las ideas, la forma de vida (Castro-Gómez et al., 2007).

Cada término ("poscolonialismo" y "decolonialidad"), con sus correspondientes diferencias, se basan en una crítica al sistema colonizador que existió y existe de una manera distinta en la actualidad, buscan explicarlo y reflexionar acerca del mismo. Sin embargo, en este sentido podríamos señalar un matiz que resalta cierta distinción entre los conceptos anteriores y el de "neocolonialismo", matiz que mencionaremos más adelante. Por el momento, es necesario mencionar brevemente el contexto teórico que está de fondo acompañando a este último término. A pesar del continuo y normal uso que se hace de esta palabra desde hace más de 50 años, no hay una definición general que la identifique, y tampoco es claro el momento de su origen. Algunos autores atribuyen su invención a Jean Paul Sartre, quien lo usó por primera vez en 1956 en uno de sus escritos. Otros ubican su origen en el Leninismo, en el que fue utilizado para describir una nueva forma de dominación impuesta después de algún periodo colonial en los estados recientemente independizados (Haag, 2011).

Vasili Vajrushev definió el neocolonialismo como "una política colonial llevada a cabo por los poderes imperialistas con mecanismos escondidos y nuevos 
para reforzar el capitalismo, maximizar el aprovechamiento y mantener la influencia económica, política, ideológica y militar de los tiempos de la colonia" (Haag, 2011, p. 9. Traducción mía). La primera definición oficial fue proporcionada por la All-African People's Conference en 1961, en la cual se dijo que el neocolonialismo es "[l]a supervivencia del sistema colonial a pesar del reconocimiento formal de la independencia política en países emergentes, los cuales devienen víctimas de una forma indirecta y sutil de dominación mediante recursos políticos, económicos, sociales, militares o técnicos" (Haag, 2011, p.9. Traducción mía). Sin embargo, el concepto solo ganó atención internacional hasta que Kwame Nkrumah publicó su libro sobre el tema que nos ocupa. De acuerdo a Nkrumah, la esencia del neocolonialismo reside en que el estado sujeto a este es oficial y formalmente independiente y soberano; no obstante, es controlado económica y políticamente desde afuera. El neocolonialismo se entiende como una forma de colonialismo adaptada, la cual se ayuda de la debilidad de los Estados recién independizados; todo con el propósito de obtener beneficios de tipo económico, político y cultural, lo que se lleva a cabo generalmente otorgando el poder político a las élites del Estado, aún dependiente, que favorezcan a los países dominantes. De cualquier forma, "la meta es la misma que durante el colonialismo; mantener las antiguas colonias en una posición de dependencia que permite la explotación económica” (Haag, 2011, pp. 9-10. Traducción mía).

Philip G. Altbach, profesor investigador y director del Center for International Higher Education en la Lych School of Education en Boston College, menciona, años más adelante, en 1971, que:

debemos usar "neocolonialismo" para designar el continuo impacto postcolonial de países industrializados y avanzados en los sistemas y políticas de educación, así como en la vida intelectual de áreas en desarrollo.

El neocolonialismo moderno no involucra control político directo y deja una libertad de acción sustancial a los países en desarrollo. Sin embargo, algunos aspectos de dominación de la nación avanzada sobre el país en desarrollo se mantienen. El neocolonialismo es, en parte, una política planeada de países avanzados para mantener su influencia en los países en desarrollo, pero es también, simplemente, una continuación de prácticas pasadas. (Altbach, 1971, p. 237. Traducción mía) 
Las maneras de continuar con la dependencia de un país son, de acuerdo con cada situación, muy sutiles y variadas. Algunas de estas son las influencias ejercidas en los sectores educativos y culturales, lo cual significa que el sistema neocolonialista se puede ver reflejado y afectar incluso a esas áreas (Haag, 2011).

Originalmente, el concepto "neocolonialismo" era utilizado para designar las prácticas que países anteriormente poseedores de colonias como Francia e Inglaterra llevaban a cabo con sus antiguos subyugados. Sin embargo, poco a poco su uso se expandió y se comenzó a emplear para describir las situaciones de dominación económica ejercidas por otras potencias como Estados Unidos de América, la Unión Soviética o China. Además, recientemente se ha llegado a utilizar para señalar la explotación ejecutada por compañías multinacionales y los programas de cooperación de organizaciones internacionales llevados a cabo en países en desarrollo. Esta gran difusión del concepto ha causado que se utilice inadecuadamente, tales circunstancias han sido las responsables de la pérdida de credibilidad que el concepto haya podido tener en los últimos 50 años (Haag, 2011).

Conocemos ahora más de una definición del término "neocolonialismo", cada una de las cuales guarda importantes semejanzas entre sí; sin embargo, es pertinente quedarnos con una sola definición que alcance a abarcar los matices que cada una de las anteriores nos brinda, además de algunas características que también definen al término y que no están contenidas en alguna de las ya mencionadas. Por esta razón, la definición que proponemos es la siguiente: se puede entender por neocolonialismo una nueva forma de colonia. Es decir, la dependencia económica, política, cultural, ideológica e, incluso, educativa, de un país con respecto a otro. Sin embargo, a diferencia del colonialismo tradicional, el neocolonialismo tiene la particularidad de que el país neocolonizado no está ocupado físicamente por fuerzas del país dominante; dicho de otra forma, no existen colonos del país invasor que, en representación de su lugar de origen, se encuentren físicamente en el país invadido para someterlo. Por el contrario, el país neocolonizado tiene independencia política de forma oficial. Existen, más bien, otros métodos para mantener la dependencia de las antiguas colonias, métodos indirectos que provocan una dominación beneficiosa en muchos aspectos para el país anteriormente invasor. Así, una de las maneras indirectas de influencia más mencionadas es que el país dominante apoye y mantenga acuerdos con las élites que tienen el poder político en los países dominados. 
Para finalizar, retomamos el punto que dejamos pendiente antes, es decir, la posible distinción que hay entre "poscolonialismo", "decolonialidad" y "neocolonialismo". Consideramos que la diferencia que hay entre estos términos es que, mientras que poscolonialismo y decolonialidad son entendidos como partes de ciertas escuelas de pensamiento, crítica y propuesta; neocolonialismo denota, en general, una situación específica. Es decir, el concepto no refiere a una crítica o no se pretende proponer con él algo, más bien, es un término con el que se busca describir cierta circunstancia, en este caso, la de la nueva forma de dominación por parte de algunos países con respecto a otros.

\section{Neocolonialismo en nuestros días}

Hemos mencionado ya lo que debe entenderse por neocolonialismo, por tanto, ahora conviene abordar los diferentes aspectos por los que unos países tienen gran influencia sobre otros. Comenzaremos mencionando el aspecto que concierne al sector educativo. Como señalamos en el anterior apartado, el fenómeno neocolonial es notorio incluso en el aspecto académico. A grandes rasgos, es pertinente decir que el sistema de educación de los países en desarrollo refleja una estructura y organización escolar de un modelo extranjero, lo cual tiene necesariamente un impacto en la naturaleza de la educación proporcionada. Muchos de los libros son importados de países avanzados y con frecuencia encontramos en escuelas y universidades maestros expatriados, de manera que el lenguaje del país dominante prevalece como medio de instrucción en el sistema de educación de países en desarrollo. Tal aspecto repercute directamente en el sistema educacional, ya que los valores que se inculcan y la posibilidad de educación que existe para distintos sectores de la población del país en desventaja mantiene el discurso dominante (Altbach, 1971).

Un ejemplo de las influencias culturales del neocolonialismo lo encontramos en los medios de comunicación, ya que estos actúan como una herramienta fundamental para fomentar el fenómeno neocolonial. Se menciona que países que ejercen esta forma de dominación han aplicado una estrategia denominada por Martín Serrano como "influencia sin asimilación" (algo que más tarde se expondrá con mayor detalle y que es fundamental en el pensamiento de Zea), dicho de otra forma: transculturización, concepto que se refiere a la evasión de los mestizajes 
de tipo cultural o biológico. Desde esta perspectiva, el autor sostiene que "el neocolonialismo es una transformación del colonialismo que fue posible gracias a las capacidades transculturizadoras de los medios de difusión masivos. Las políticas coloniales y comunicativas se han entreverado" (Martín Serrano, 1977, p. 193).

Además, Serrano menciona que esta forma de control ha conseguido dos cosas que ninguno de los anteriores imperios pudo lograr con los medios con los que contaba: "controlar a distancia las culturas de las comunidades dependientes; y borrar la distinción entre las intervenciones que producen beneficios económicos y políticos y las que promueven la transculturización" (Martín Serrano, 1977, p. 193). El proceso en el que se crea la dependencia puede ocurrir de la manera siguiente: en los medios de comunicación masiva se popularizan canciones, películas y otros materiales de multinacionales de la industria cultural. Los medios de difusión, al ser dependientes de la publicidad, programan su información y entretenimiento de acuerdo con el producto o servicio extranjero en boga. Una vez que estos productos se han implantado en el mercado, se produce una dependencia comercial, tecnológica y cultural de esos bienes.

En el aspecto económico y político se debe mencionar que las élites capitalistas que se encuentran en un país en desarrollo son de gran ayuda a las élites transnacionales para asegurar la posición imperialista de las últimas. Las élites locales le garantizan a las potencias imperialistas una estabilidad económica y la ejecución de reformas estructurales favorables a la continuidad del sistema dominante. Los Estados imperiales, entonces, apoyan a instituciones financieras internacionales, pues les sirven de herramienta de penetración, dominación y control de los Estados neocoloniales, los cuales se subordinan a sus dictados, siendo así una garantía de defensa de los intereses de capitales transnacionales:

La nueva estructura neocolonial del Estado se organiza para la transferencia de valores y recursos a las metrópolis donde se ubican las grandes corporaciones transnacionales (...) A diferencia del trabajo, el capital transnacional no se encuentra regulado, pero actúa sobre aquél mediante arreglos institucionales que lo sujetan al control directo de los Estados nacionales.

Esto da origen a un Estado neo-colonial que es activo, regulador e intervencionista (...) [del que] sus actividades, reglas e intervenciones se orientan a servir los intereses del capital extranjero y de la clase capitalista transnacional. (Vargas Hernández, 2005, pp. 1-2) 
Para finalizar este apartado, abordaremos algunas regiones latinoamericanas que han estado sujetas por muchos años al fenómeno neocolonial. Raymond $\mathrm{E}$. Crist realizó un estudio comparativo desde el año 1928 al año 1982 en Venezuela, con el objetivo de recoger datos acerca del ambiente cultural de la zona. En su estudio se percató de que la tierra tenía gran importancia mercantil en este lugar y que estaba concentrada en las manos de unas pocas familias, las cuales la poseían por "derecho de antigüedad"; es decir, porque desde años atrás tales tierras habían sido de su propiedad, y no por el derecho legal que les permite ser dueños de ellas. Los trabajadores de bajos recursos, que no tenían tierras, limpiaban sus parcelas en las áreas arboladas y ahí sembraban lo más necesario. Los más favorecidos económicamente criaban ganado, cosechaban caña de azúcar, algodón y sésamo para las industrias, de forma que podían obtener grandes créditos o subsidios. Los granjeros con menos posibilidades obtenían solo unos pocos o nada de créditos y casi nada de asistencia técnica para la mejora de su pequeño negocio, de forma que les era imposible muchas veces hacer llegar su producto al mercado (Crist, 1987).

No solo en países como Venezuela y México, sino también en Filipinas, Jamaica, Puerto Rico, Guatemala y Ecuador, muchos de los granjeros que cultivaban en fértiles suelos aluviales diversidad de alimentos para consumo personal y local, fueron desplazados y negados del acceso a la tierra o al mercado de la misma. El fenómeno de la concentración de la tierra en pocas manos fue sucediendo de manera rápida. Por esta razón, Crist (1987) sostiene: "En una moda de capital intensivo, altamente mecanizada, se trabaja en producir cultivos para exportar por compañías responsables del interés de ningún país. Este es el patrón del neocolonialismo [traducción propia]" (p. 204).

En el caso de África, tenemos un estudio más reciente, hecho en el año 2013, en el que se explica, con referencia a los estándares globales desde el año de 1960, que el África subsahariana ha logrado un escaso e insignificante crecimiento por sí sola. Este crecimiento solo ha beneficiado a unos pocos a costa de muchos, como en el caso latinoamericano.

Indicadores de desarrollo como el de la pobreza, la desigualdad y el desempleo señalan niveles bajos de calidad de vida, empeorando durante el periodo ya mencionado. El aumento de inversión extranjera pareciera ser la solución a la situación económica de esta región; sin embargo, la mayoría de estas inversiones 
van hacia industrias que están manejadas por compañías extranjeras o, en otros casos, por unos pocos hombres y mujeres africanos que se dedican al negocio (Watson, 2013).

Siguiendo esta la línea de reflexión, cabe mencionar un dato que se explica en uno de los estudios revisados y que podría sumarse a las manifestaciones del régimen neocolonialista en el aspecto cultural y rutinario. Se comenta que los patrones del neocolonialismo se encuentran hasta en el trato diario con las personas y la actitud corporal que se adopta hacia las mismas. Se expone que en la actualidad los descendientes de los colonos que llegaron a África, a pesar de que tienen la intención de ayudar a los pobladores originarios de esas tierras, quieren diferenciarse constantemente de los mismos, manteniéndose en un lugar especial que los distinga: el de "salvadores". Para explicar esto, los estudios exponen un ejemplo muy cotidiano, tomado de un caso real, en el que se muestra esta actitud de mantener la distancia. Se comenta que estando tres hombres de origen africano y una mujer extranjera listos para trabajar bajo la sombra de un árbol, deciden detenerse a tomar un poco de agua para calmar su sed. Los hombres beben todos del mismo vaso, mientras que la mujer busca marcas y limpia el vaso frente a ellos para cuidar de no tocar sus labios o beber agua del mismo sitio del que habían bebido los otros. Al preguntarles a los hombres africanos qué pensaban de este hecho, ellos mencionaron: "Verás: ella tiene apartheid en el corazón" (Watson, 2013, p. 3. Subrayado y traducción míos).

\section{El neocolonialismo en el pensamiento de Zea}

El PROPÓSITO DEL PRIMER APARTADo fue ubicar el término "neocolonialismo" en un marco histórico-teórico de forma que pudiésemos diferenciarlo de otros conceptos importantes como "decolonialidad" y "poscolonialismo" y así, finalmente, otorgarle una definición propia útil para comprender y delimitar la problemática a la que nos referimos aquí. A continuación, trataremos el tema del neocolonialismo desde la perspectiva de Zea, por tal motivo, nos serviremos de la definición de la sección primera; de manera que cuando nos refiramos al neocolonialismo en la propuesta de Zea, debe entenderse por él lo ya señalado en un principio. Es importante aclarar que a pesar de que el filósofo mexicano no nos otorga explícitamente una acepción de neocolonialismo tal como nosotros la he- 
mos postulado, consideramos que la definición que brindamos encaja en buena medida con las ideas y posturas de Zea e incluso podríamos inferirla de las mismas. Además, sostenemos que esta manera de entender el neocolonialismo conecta tanto prácticas actuales y pasadas del dominio neocolonial, ya que funciona como una definición general que considera diversos aspectos de ellas y es común a ambas.

Por tanto, para introducirnos de lleno en este segmento del escrito, es conveniente comenzar mencionando que Leopoldo Zea es uno de los filósofos latinoamericanos más destacados, cuyos pensamientos fueron de gran influencia en polémicas de discusión filosófica, política, social, etc. que tienen repercusión e importancia hasta el día de hoy. Una de ellas es la que comprende el tema del neocolonialismo, nuestro punto central de discusión. Sin embargo, con el fin de entender con mayor claridad y de forma más completa las ideas del filósofo, es necesario ser específicos, de manera que señalemos cuáles de estas ideas son las que a lo largo de su carrera intelectual se relacionan más profundamente con el tema que nos ocupa y tienen importantes aportaciones. A diferencia de como sucede con otros autores, en el caso de Zea no es posible ubicar, con el fin de hacer más clara nuestra comprensión de su propuesta, un "primer" o un "segundo" periodo de pensamiento.

La razón por la cual no nos inclinamos por tal categorización del pensamiento de Zea es porque su trayectoria intelectual evidencia que esta ha sido más o menos constante y lineal. Es decir, durante su trayecto, el filósofo se interesó por ciertos temas que mantuvo y fue desarrollando a lo largo de su carrera conforme iba ganando experiencia. Además, no es fácil percibir giros importantes en su pensamiento o cambios de perspectiva significativos que lo hicieran desdecir lo que sostuvo en algún momento. Dado lo anterior, consideramos que referirnos a un primer o segundo Zea para establecer el contexto en el que nos situaremos al analizar sus ideas, no era la mejor opción. Sin embargo, esto no excluye que haya algún lector exigente que demandase periodos de tiempo específicos; si este fuese el caso, entonces se debe considerar que estamos analizando la producción intelectual de Zea durante las décadas de los años 60 y 70, a excepción de una entrevista realizada en 1998.

Hecha esta aclaración, proseguiremos a cifrar algunos de los temas a los que Zea les dedicó más atención durante su trayectoria intelectual. En primer lugar, la unión latinoamericana. $\mathrm{Al}$ respecto, el filósofo defendió la alternativa de una 
unión multifacética entre los países latinoamericanos, no como utopía, sino como un proyecto acorde con el devenir histórico, teniendo en mente el sueño bolivariano. Además, sostuvo la existencia de un pensamiento auténtico latinoamericano, la producción de una filosofía propia y la posibilidad de que tal pensamiento se insertase en el panorama como universal (Conaculta, 2010). Expuso sus propuestas desde una perspectiva historicista, por lo que resaltaba la importancia de la comprensión histórica. La relevancia que brindó Zea al papel de la historia se reflejó igualmente en conceptos que desarrolló con profundidad, como el de "asimilación”, el cual está directamente ligado a la capacidad de comprender el pasado (Villegas, 1956). Asimismo, entre sus principales preocupaciones estuvo el desarrollo del concepto de "toma de conciencia", clave para entender muchas de sus ideas. De igual forma, el análisis de la situación neocolonialista de muchos países fue una de sus reflexiones más profundas (Zea, 1971). Finalmente, cabe mencionar que durante su carrera no se limitó a contribuir en el terreno teórico; él creía en que las ideas debían ser llevadas a la práctica y que las acciones eran necesarias para generar un cambio, por lo que estaba a favor de organizar una política de la cultura que hiciera de las ideas motores de servicio a la comunidad (Conaculta, 2010).

De este modo, para abordar el tema del neocolonialismo desde la perspectiva de Zea, es necesario, en primera instancia, tener presente que cuando el autor piensa en neocolonialismo se refiere a cierto protagonista que juega, sino el más importante, uno de los papeles más relevantes en el acontecer del fenómeno neocolonialista: el dominio de Estados Unidos de Norteamérica. Al respecto Zea (1971) sostiene:

la experiencia latinoamericana es una experiencia importante para la historia del mundo contemporáneo, del mundo en que vivimos. La experiencia de pueblos que se enfrentaron a la expansión y a la hegemonía de la poderosa nación [Estados Unidos] que al principio de este siglo XX planteó la necesidad de un nuevo reparto del mundo, esto es, de un reparto en que pudiese participar, ya que el mundo entero se encontraba repartido. Un nuevo reparto y, también, una nueva forma de colonización, un neocolonialismo al que acabarían subordinándose no sólo las antiguas colonias de Europa Occidental, sino los mismos imperios que habían participado en el primer reparto y ahora eran parte del botín por repartir (p. 8. Subrayado mío). 
Estados Unidos es, entonces, visualizado como uno de los principales responsables de que el neocolonialismo aparezca en el panorama como nuevo sistema de organización social, económica, política, etc. Específicamente, Zea concibe este sistema como una forma de seguir manteniendo la dependencia del colonialismo, pero mediante nuevas formas de sometimiento tanto en los planos político, económico y cultural, tal como explicamos con más detenimiento en el primer apartado. Para sostener esto, Zea no solo toma en cuenta el caso de México, sino que también tiene en mente a países de Asia y África, pues para él, estos padecen la misma situación que Latinoamérica.

Apenas acaba Latinoamérica de liberarse políticamente de España y Portugal, todavía se debate por emanciparse mentalmente de los hábitos y costumbres que le impusiera la colonia, cuando se ve enfrentada a resistir o a someterse a un nuevo imperialismo, el que nace en América del Norte. Una experiencia en la que participaran otros pueblos del mundo que, como los latinoamericanos, se sacuden los arreos que les han impuesto los imperios surgidos en Europa occidental. Los pueblos de Asia y África, como los latinoamericanos, aún no se libran plenamente del colonialismo que les impusiera Inglaterra, Francia, Holanda y otras naciones europeas, cuando se tienen que enfrentar al imperialismo, el neoimperialismo ${ }^{1}$ al que vienen enfrentándose los pueblos de Latinoamérica desde los inicios del siglo XX, los Estados Unidos. (Zea, 1971, p. 8)

Zea resalta que esta forma de convivencia entre naciones se sostiene a costa de la explotación de unos para favorecer a otros, tal como sucede con los pueblos latinoamericanos y algunos de Asia y África, los cuales son, según Zea, pueblos marginales, ya que sirven como instrumentos de un progreso que no les pertenece. Estados Unidos convierte sus fines en los fines de los demás, y su propio desarrollo, en la meta del desarrollo de las naciones dependientes, por lo que se podría decir que "[1] a grandeza de unos pueblos la hacen la audacia de unos (...) y la miseria de otros” (Zea, 1971, p. 35). Muchos países latinoamericanos trataron de seguir su ejemplo, poniendo como meta crear una civilización moderna como la de aquellos; sin embargo, resultó que tal "civilización (...) se reducía a la incorporación de estos pueblos al neocolonialismo” (Zea, 1976, p. 196). Desde

1 Zea no establece una diferencia entre los conceptos de neocolonialismo y neoimperialismo. 
la perspectiva del filósofo, simplemente se adoptaron métodos más eficaces para la ya antigua explotación de la tierra.

Zea es consciente de que dicho sistema neocolonialista no podría mantenerse si no existiera la mutua cooperación entre los países que lo ejercen y algunos cuantos interesados que residen en el país dominado. Es decir, existe una alianza y una convivencia de los intereses de las potencias como Estados Unidos y los grupos conservadores o las élites del país neocolonizado, quienes temen un cambio de orden que pudiese transgredirlos. De hecho, ciertos males de las naciones en desventaja actúan como instrumentos de preservación de la hegemonía de la potencia sobre el resto de los países menos favorecidos. "Surgen testaferros y dictadores [en los países dominados] encargados de mantener el orden que mejor conviene a la expansión y desarrollo del nuevo imperialismo" (Zea, 1971, p. 16).

Uno de los efectos del neocolonialismo es que los habitantes de un lugar adquieren costumbres, hábitos, maneras de pensar, ideales, expectativas de vida, creencias, gustos, ambiciones, etc. muy similares a los del país que los domina. Podría decirse, entonces, que también existe una neocolonización cultural. Se pierden de vista los elementos que pudiesen construir y constituir una identidad propia y, por otro lado, se imitan los de la cultura dominante, aspirando a una forma de vida idealizada y ajena al propio entorno. Tal es el caso del vínculo que existe entre México y Estados Unidos. Zea menciona que hay una relación confusa entre estos dos países; ya que debido a la admiración mexicana hacia la prosperidad y progreso que Estados Unidos ha alcanzado habiendo sido una colonia de Inglaterra, Estados Unidos ha sido puesto como ejemplo a seguir y modelo a imitar, por lo que los mexicanos han querido adoptar muchas de sus estructuras de organización e ideales. Sin embargo, al mismo tiempo, Zea piensa que esa admiración fue menguándose debido a la manera en la que Estados Unidos ha alcanzado dicha prosperidad, es decir, ocupando el vacío de poder que dejaron las antiguas colonias; además de que es una nación que, tal como hacían las anteriores, le usurpa "la humanidad" a los habitantes de las naciones sometidas como las de América Latina (Villegas, 1956).

No obstante, el fracaso al tratar de acabar con guerras, anarquías, revoluciones, etc., adoptando las costumbres, técnicas, ideales, estructuras y hábitos de la nación que había logrado la emancipación, lo explica Zea por medio de la diferenciación de la forma de ser de cada pueblo. Los estadounidenses son idealistas 
prácticos y los mexicanos, así como otros que se hallan en su situación, prácticos idealistas (Villegas, 1956). Zea comenta que los primeros tratan de adaptar sus ideas a la realidad, mientras que los segundos pretenden adaptar la realidad a sus ideas; los primeros adquieren seguridad y alcanzan el éxito, los segundos consiguen frustraciones y fracasos. Por lo tanto, resume el filósofo que para convertirse en Estados Unidos no basta con trasladar sus normas, instituciones, reglas y forma de vida. En realidad, la búsqueda tanto de una identidad como de la propia emancipación y prosperidad debe buscarse en y desde la propia circunstancia y con los esfuerzos propios, aclara Zea (Zea, 1971).

A partir de este punto, comenzaremos concretar algunas de las ideas y propuestas de Zea, las cuales son una posible salida, alternativa de reflexión y crítica, a la situación del neocolonialismo. En un panorama en el que se opta por la imitación y adopción de ideas y formas de proceder ajenos, Zea apuesta por la autenticidad, la cual puede encontrarse potencialmente en cualquier raza y con la que se puede resolver los problemas que a cada pueblo le conciernen; es factible para quien desea "volverse protagonista de su propia historia provisto de las armas de la razón, la democracia y la libertad” (Gómez Martínez, 2010, p. 260). Zea enfatiza que Latinoamérica, por ejemplo, posee una historia que es solo suya y, además, ha adquirido una cultura y organización social que la identifican, hechos a partir de los cuales cualquier pueblo debiese fomentar su propia emancipación. $\mathrm{Ni}$ Estados Unidos ni cualquier otra nación puede negarle la humanidad a quien ha nacido humano; por el hecho de serlo, debe ser reconocido como tal. En consecuencia, los habitantes de los diferentes pueblos cuentan con la facultad de ser ordenadores de su propia realidad y capaces de realizarse en su propia circunstancia. Ser humano, entonces, no significa ser la copia de otro algo o alguien, sino tener una personalidad propia.

Según Zea, para ser capaz de realizar esto no basta con romper el vínculo político directo con la Corona española o portuguesa. En el caso de los latinoamericanos se necesita romper con la forma de vida heredada de estos, es decir, alcanzar una emancipación mental. El filósofo resaltaba que: "Latinoamérica, emancipada políticamente de sus metrópolis, no ha podido, pese a los esfuerzos de sus élites liberales, emanciparse de los hábitos y costumbres que le impusiera la Colonia”' (Zea, 1971, p. 81). Por lo anterior, se debe buscar la formación de un 
criterio crítico que cuestione la cultura heredada con el fin de asumirla y hacer lo que sea necesario para vivir auténticamente.

Hacerse dueño de la propia circunstancia, como mencionábamos en un párrafo anterior, implica tomar en cuenta el pasado de forma especial. Es decir, haciendo referencia a la perspectiva histórica que caracteriza su pensamiento, Zea considera que es necesario aprender de los errores del pasado, tales como la negación de la humanidad del latinoamericano o de su capacidad de pensar para crear estructuras, se debe tomar conciencia de que no se tiene por qué ser imitación de otros pueblos (Gómez Martínez, 2010). Dicho aprendizaje es esencial para una buena comprensión histórica, la cual implica saber dónde están situados en el presente los hechos del pasado y, también, las razones por las que estos sucedieron; todo con el propósito de que ciertas situaciones no se repitan: "[e]n otras palabras, cuando cada hombre actúa no puede ser precedido por el pasado (...) el individuo debe tener la capacidad de asumir la responsabilidad del pasado, porque solo de esa manera podrá afrontar el presente" (Colonna, 2012, p. 24). Dicho lo anterior, es pertinente introducir ahora el término de "asimilación", ya que este está directamente relacionado con lo explicado previamente. Asimilar, por tanto, consiste en dejar de lado y olvidar nuestra historia, es decir, es posible y conveniente negar el pasado, pero habiéndolo asimilado como parte de nuestra historia, habiéndolo concientizado, dejándolo en la categoría de experiencia vivida y conocida, y apuntando, con esto, a dar un paso adelante y evitar cometer los mismos errores (Villegas, 1956). Empero, la asimilación también puede ser entendida como "hacer propio lo que parecía extraño acomodarlo a lo que se es, sin pretender, por el contrario, acomodar el propio ser a lo que le es extraño" (Zea, 1960, p. 9).

Zea propone una actitud de asimilación ante las ideas que vienen del extranjero. No considera que sea perjudicial para un país tener apertura ante la cultura e ideología de otros que han logrado posicionarse en el panorama internacional. Sin embargo, piensa que no se debe solo imitar, sino asimilar. Es decir, se debe pensar desde la situación particular en la que nos encontramos, concientizar el pasado del que procedemos y el presente en el que vivimos y nos afecta, de manera que repensemos las ideas que vienen de fuera contextualizándolas en el ambiente cultural, político, económico, etc. que nos pertenece. De esta forma, existirá un pensamiento analizado y estructurado de acuerdo con nuestro propio contexto, adoptando una actitud de independencia, de distanciamiento, de críti- 
ca ante todo aquello que llega de afuera, generalmente impuesto sin ser cuestionado (Gómez-Martínez, 1988).

Por otro lado, el filósofo apela constantemente al concepto de "toma de conciencia”. Para él, el tener conciencia implica complicidad entre yo y el otro, implica, también, que se tiene conciencia de él y de su humanidad. No es fácil llegar a dicho estado, pues generalmente el otro se encuentra en medio de una serie de afirmaciones y negaciones; dicho de otra forma, cada persona le niega su humanidad a la otra al tratar de cosificarla pero, al mismo tiempo, pretende afirmarse al exigir del otro el reconocimiento de su propia humanidad. Según Zea, la "toma de conciencia" era una característica de su época, la cual significa saber que no estamos solos sino que, constantemente, estamos con otros, los cuales son nuestros semejantes; sin embargo, al aceptar esta semejanza deberíamos estar dispuestos a aceptar también la diferencia: la diversidad (Zea, 1971).

El rechazo a la diferencia o la no aceptación de la diversidad es, según el filósofo mexicano, una de las causas principales del neocolonialismo. Es decir, se puede entender que el otro sea distinto, que viene de otro lugar, que tiene otras costumbres, que posee otra forma de organización social o religiosa, pero como no se le suele aceptar como semejante, se le considera, entonces, inferior por ser diferente. Lo que se pretende es "absorberlo" y enseñarle lo que "debe de ser" de acuerdo con otra concepción de vida, de mundo, de cultura y de sociedad (Gómez-Martínez, 1988).

Zea, al mencionar el caso de Estados Unidos, dice que esta es una nación que se independizó de su dominio británico y que concibió para ella misma un sistema de valores que versan sobre la libertad y la dignidad del hombre. Sin embargo, estos valores no tenían pretensión de universalidad, solo eran aplicables a los ciudadanos que perteneciesen a aquella nación. Al respecto Zea (1971) menciona:

Dentro de los valores enarbolados por el Occidente está el que se refiere a la dignidad del hombre con todas sus implicaciones. Salvo que esta esencia no es reconocida sino entre sus propios creadores. Fuera de los mismos, los occidentales, se resisten a reconocer signo alguno de humanidad. La Humanidad parece agotarse en el mundo occidental y sus herederos, los Estados Unidos. (p. 30)

Un país se emancipa y controla a los demás partiendo de la idea de que deben adaptarse a la visión que el anterior tiene. En palabras de Zea (1971): 
Los Estados Unidos no sólo se emancipan del mundo occidental sino que invierten los términos y se transforman en su líder. Líder, no ya de ideales y principios, sino de su espíritu de dominio. Un dominio que revertirá sobre sus propios creadores europeos occidentales, sometiéndolos al nuevo orden occidental, ahora bajo el predominio estadounidense. El líder de la emancipación se convierte en líder del dominio. (p. 32)

Zea brinda una respuesta a esta complicada disputa y, con ello, una posible propuesta, en la que se exige la aceptación del propio ser pero se rechaza el ser del otro. El filósofo dice que esta pugna referente a la negación de la diversidad termina cuando se hace una conciliación. La síntesis de la dialéctica se da cuando nos reconocemos en la diversidad de esos otros, en su ser hombres sin más. Cuando nos sentimos plenamente con los demás, y cuando no exigimos para los demás lo que no estamos dispuestos a aceptar para nosotros mismos (Zea, 1971, p. 28).

Asimismo, en este sentido, Zea propone la igualdad en la diferencia, igualdad porque todos nos reconocemos en nuestra humanidad y en nuestra diferencia como algo que nos es común, y donde consideramos un derecho el ser diferentes. Aceptar el principio que nos propone el filósofo, implicaría el mejoramiento de las relaciones entre muchas de las distintas naciones, pues "al concebir la diferencia como un derecho, lejos de excluir, propiciaría las condiciones ideales en donde todos podrían participar en un diálogo auténticamente universal" (Montalvo, 2012, p. 30).

Mencionábamos en párrafos anteriores que Zea no solo contribuyó con propuestas teóricas, sino que también exhortaba a la ciudadanía a llevar a cabo acciones concretas para efectuar el cambio. Una de esas propuestas es la de la unión de los países latinoamericanos. Zea invita a una integración por la libertad; propone que los países latinoamericanos que comparten un pasado muy cercano y común sean conscientes de su origen histórico y de sus parecidos culturales, de forma que busquen un acercamiento mutuo. El filósofo piensa que esta integración, de tipo político y económico, por ejemplo, es una respuesta a la fuerte dependencia que existe de un país con respecto a otro, como en el caso de Estados Unidos y diversos países latinoamericanos. De hecho, Zea (1977) menciona: "lo que hoy se habla es precisamente de buscar la integración, pero bajo la libertad. Así como fuimos integrados bajo la dependencia” (p. 310). 
Para finalizar este apartado, comentaremos una última propuesta, también de naturaleza práctica, de nuestro autor. Zea se cuestiona si Latinoamérica, Asia o África, podrán llegar a tener la misma prosperidad económica de la que goza Estados Unidos y, así, emanciparse. En primer término, nuestro autor aclara que no será suficiente la asimilación de cualquier filosofía o la simple copia de ciertas legislaciones que pertenecen a países extranjeros. Sin embargo, tomando en cuenta las pasadas experiencias, concluye que aquellos países que han logrado emanciparse, como Estados Unidos, lo han hecho porque han utilizado a países en desventaja para obtener su progreso. Zea señala que Latinoamérica no podría emplear la misma táctica porque no cuenta con ese recurso (el de un país en desventaja), nosotros somos el continente dominado y explotado. Por tanto, si queremos la emancipación, Zea piensa que nosotros mismos seamos el continente que explotemos y sobre el que tomemos ventaja, ya que debemos encontrar la manera de abastecernos mediante nuestros propios recursos.

Ninguna otra nación podrá hacer fincar su progreso y desarrollo en la riqueza y el trabajo de pueblos que han sido ya transformados en cotos privados de determinadas potencias (...) Esto es, no existe ya pueblo alguno en toda la faz de la tierra que haga por Latinoamérica lo que Asia, África y la misma Latinoamérica han hecho por el llamado mundo occidental. (Zea, 1977, p.310)

\section{Respuestas finales}

Hemos Visto EN LOS APARTADOS ANTERIOREs las ideas más actuales y las de Zea con respecto al tema del neocolonialismo. Basándonos en ellas, brindaremos las respuestas a las preguntas formuladas al inicio del artículo. Las preguntas son: ¿Es la posición de Zea respecto a este tema obsoleta en cuanto a la situación que vivimos actualmente muchos habitantes en este como en otros países del mundo sujetos a un dominio neocolonial, o es pertinente considerarla seriamente como una alternativa de crítica, pensamiento e, incluso, de iniciativa de acción para tratar con la situación neocolonialista que muchos países padecen? ¿Cuáles son las razones para sostener lo anterior?

A la primera pregunta, la respuesta es que las propuestas de Zea no son en absoluto obsoletas; por el contrario, tienen total pertinencia y son dignas de retomarse para la reflexión sobre una situación neocolonialista que existe hasta el 
momento. La razón por la que sostenemos lo anterior, es que si analizamos un poco los apartados previos, podremos darnos cuenta de que el neocolonialismo estudiado en la actualidad y el estudiado por Leopoldo Zea tienen muchos elementos en común. Prácticas que tenían lugar en el periodo histórico de Zea siguen sucediendo en nuestros días. Simplemente, recordando lo ya expuesto, podremos percatarnos de que las distintas posturas, las actuales y la de Zea, coinciden en que el neocolonialismo es un fenómeno multifacético que se sostiene en la existencia de la supremacía de una nación con respecto a otra; de manera que la miseria, el subdesarrollo o la dependencia de un país asegura la prosperidad y avance de otro. Asimismo, tal como nuestro autor menciona, este sistema se mantiene gracias a que las élites del país dominado apoyan los propósitos de las potencias, situación que expusimos en la primera sección de este artículo, como una circunstancia que sigue presentándose en la actualidad.

Además, se menciona que el control que ejerce un país sobre otro puede manifestarse en la cultura del país sometido, en su forma de vida, en sus aspiraciones y en sus ideas. De hecho, no sirve de mucho tratar de resolver los problemas de índole práctico de una nación sometida bajo un sistema neocolonial, si las estructuras mentales de esta siguen estando influenciadas fuertemente por el país dominante. Vemos, entonces, que la situación económica, académica, cultural y social, que tienen algunos países de Latinoamérica, Asia y África, en general no ha cambiado mucho, tal como se menciona en el primer apartado, por lo que conserva la misma dinámica a la que Zea hace alusión cuando se refiere a la miseria que unos países deben pasar a favor del progreso de otros. Bien señala Zea que esta es la nueva forma del antiguo colonialismo traído al panorama por el nuevo imperio: Estados Unidos.

Ya que las consideraciones que Zea tiene sobre el neocolonialismo de su época, y las circunstancias que se han dado en torno a este asunto en nuestros días se relacionan directamente y tienen muchas similitudes; es importante retomar las propuestas del autor, pues responden a la situación neocolonialista actual que, a pesar de no ser exactamente la misma que la del tiempo del filósofo, se ha mantenido vigente en su forma básica y guarda un profundo parecido con la visión que Zea tiene sobre ella. Tal como lo dijo Germán Arciniegas: "Zea no es un pensador del ayer, ni de hoy, sino del mañana” (Saladino, 2012, p.11, cita a Arciniegas en Zea, 2003). 
En la sección anterior enunciamos varias de las propuestas de Zea, entre las que tenemos algunas de índole práctico y otras de carácter puramente teórico. Expusimos la propuesta por la autenticidad de pensamiento, en la que se busca una emancipación no solo en el ámbito económico o político, sino también en el intelectual. Además, comentamos sobre la asimilación del pasado y la comprensión histórica como estrategias de prevención contra la repetición de errores anteriores, que nos han traído a la situación en la que nos encontramos. Asimismo, postula la toma de conciencia, es decir, la aceptación del ser del otro, la concientización de su humanidad y al mismo tiempo el respeto por su diferencia. Finalmente, expusimos dos propuestas prácticas, una que consiste en la unión de los países latinoamericanos en la libertad y tolerancia, y otra que radica en señalar que para que Latinoamérica pueda emanciparse económicamente, debe hacerlo con sus propios recursos.

Cada una de estas propuestas, algunas más que otras, aún tienen mucho por aportar a las reflexiones que se hacen sobre la situación neocolonialista actual; propuestas que se refieren al respeto a la diferencia, a la dignidad humana y a la comprensión histórica, como lo son las de Zea, han sido fundamentales en la historia del pensamiento y han sido constantes respuestas a problemas que se han presentado a lo largo de la historia de la humanidad, cuando los derechos básicos de un individuo se han visto rebajados o transgredidos por otro que está en una situación privilegiada. Las propuestas prácticas deberían ser analizadas con más detenimiento; sin embargo, en principio consideramos que pudiesen funcionar como base para iniciativas de acción que generen cambios sustanciales.

\section{Conclusiones}

ES NECESARIO DARLE UN VISTAZO a la situación económica, política y cultural en la que nuestro continente se encuentra para percatarnos de que vivimos en un lugar que padece de un dominio neocolonialista. $Y$ dado que seguimos en tal situación, es necesario que esta se siga analizando de acuerdo con las modificaciones que presenta conforme pasa el tiempo; así como es importante que se piense en una propuesta para el problema. Por consiguiente, consideramos que el pensamiento de Zea es una de las posibles alternativas pertinentes de reflexión 
para buscar soluciones a una situación que nos afecta a todos y por la que regimos muchas de nuestras actividades día a día.

Cabe mencionar que en una entrevista para el periódico Reforma, en 1998, Zea expone sus ideas sobre el futuro de América Latina. Desde su punto de vista, este podría ser positivo o catastrófico, lo que dependería mucho de cómo las sociedades se comportarían en ese momento ante la realidad que se les presentaría. En cuanto a lo anterior, él se muestra con un espíritu de esperanza, deseando un mejor porvenir a las naciones en desventaja. Confía en que estas actúen comprometidas y responsables, no solo como simples herramientas de explotación. Sin embargo, deja abierta la posibilidad de que no se den las cosas de la manera más deseada, resultando en una anarquía absoluta donde todos habremos fracasado por el hecho de no ser capaces de convivir en otra relación que no sea la de dependencia. De modo que Zea deja abiertas ambas posibilidades. Ahora, es nuestro turno seguir analizando qué ha sucedido desde ese momento y reflexionar en cuál de los dos caminos estamos situados, hacia dónde vamos y, sobre todo, hacia dónde queremos ir.

Referencias

Altbach, P.G. (1971). Education and Neocolonialism: A Note. Comparative Education Review, pp. 237-239.

Collins Dictionary. Post-colonial. Consultado el 10.01.14. Disponible en línea: http://www.collinsdictionary.com/dictionary/english/postcolonial?showC ookiePolicy $=$ true

Colonna, R. (2012). Responsabilidad y compromiso en el pensamiento de Leopoldo Zea. Cuadernos Americanos, 141, pp. 19-28.

Conaculta. (2010). Leopoldo Zea, filósofo de la unidad latinoamericana. Consultado el 01.01.14. Disponible en línea: http://www.conaculta.gob.mx/detalle-nota/?id=5610\#.U6y-spR5Mbt.

Crist, R.E. (1987). The Pattern of Neocolonialism. American Journal of Economics and Sociology, 46(2), pp. 203-204.

Diccionario de la Real Academia Española. Descolonización. Consultado el 09.01.14. Disponible en línea: http://lema.rae.es/drae/?val=descolonizaci\%C3\%B3n. 
Gómez Martínez, J.L. (1988). La crítica ante la obra de Leopoldo Zea. Anthropos. Revista de Documentación Cientifica de la Cultura, 89, pp. 36-47.

Gómez Martínez, J.L. (2010). Leopoldo Zea (1912-2004): Igualdad en la diferencia: el ser humano como problema. Utopía y praxis latinoamericana, 15(51),pp. 5-7.

Haag, D. (2011). Mechanisms of Neocolonialism: Current French and British in Cameroon and Ghana. Barcelona: Institut Català Internacional Per la Pau. Consultado el 10.01.14. Disponible en línea: http://www20.gencat.cat/docs/ icip/Continguts/Publicacions/WorkingPapers/2011/Arxius/WP\%20 2011-6\%20ING.pdf.

Martín Serrano, M. (1977). El colonialismo se convierte en neocolonialismo cuando logra integrar el comercio y la transculturización en una única red (resumen). Revista de filosofía de la Universidad de Costa Rica, 114-115, pp. 309-312.

Martín Serrano, M. (2009). La Teoría de la Comunicación, la vida y la sociedad. Intercom. Revista Brasileira de Ciências da Comunicação, 32(1), pp. 245-257.

Mignolo, W.D. (2007). El pensamiento decolonial: desprendimiento y apertura. S. Castro Gómez \& R. Grosfoguel. (Coords.), El giro decolonial. Bogotá: Siglo del Hombre Editores.

Montalvo Piedra, P. (2012). Leopoldo Zea: entre la igualdad y la diferencia. Cuadernos Americanos, 141, pp. 29-43.

Oxford Dictonary. Postcolonial. Consultado el 13.01.14. Disponible en línea: http://www.oxforddictionaries.com/definition/english/postcolonial?q=post+ colonial.

Saladino García, A. (2012). Leopoldo Zea polemista. Cuadernos Americanos, 141, pp. 11-18.

Vargas Hernández, J.G. (2005). Neocolonialismo, resistencia, crisis y transformación del estado. Revista internacional de ciencias sociales y humanidades, 15(2),pp. 155-183.

Villegas, A. (1956). Reseñas bibliográficas. Diánoia, 2(2), pp. 386-389.

Watson, M.D. (2013).The Colonial Gesture of Development: the Interpersonal as Promising Site for Rethinking Aid to Africa. Africa today, 59(3), pp. 3-28. Young, R.J.C. (2006). ¿Qué es la crítica poscolonial? Consultado el 14.01.14. Disponible en línea:http://robertjcyoung.com/criticaposcolonial.pdf. 
Young, R.J.C. (2008). Postcolonialism: An Historical Introduction. United Kingdom: Blackwell Publishing.

Zea, L. (1960). La filosofía americana como filosofía sin más. Consultado el 10.01.14. Disponible en línea: http://www.olimon.org/uan/zea.pdf.

Zea, L. (1971). Latinoamérica: emancipación y neocolonialismo. Caracas: Tiempo Nuevo.

Zea, L. (1976). El pensamiento latinoamericano. Barcelona: Ariel.

Zea, L. (1977). Entrevista a Leopoldo Zea. A.L. Marín (Entrevistador).

Zea, L. (1998). Entrevista/Leopoldo Zea/La identidad universal. M.Á. Muñoz (Entrevistador).

Zea, L. (2003). Agradecimientos por los homenajes que he recibido al cumplir 90 años. A. Saladino \& A. Santana. (Coords.), Visión de América Latina: homenaje a Leopoldo Zea (pp. 541-542). México D.F.: FCE/UNAM/ $\mathrm{UAM} / \mathrm{IPGH}$. 